\title{
Robust attitude tracking control scheme for a multi-body spacecraft using a radial basis function network and terminal sliding mode
}

\author{
CHANGQING YUAN Y $^{\dagger}$, YANHUA ZHONG, JINGRUI ZHANG ${ }^{\S}$,
} HONGBUO LI"**, GUOJUN YANG ${ }^{\dagger}$ and YING SHEN ${ }^{\dagger}$

${ }^{\dagger}$ Aviation University of Air Force, Changchun 130022, P. R. China

Email: ycq02@mails.tsinghua.edu.cn; yanggj@163.com; shenying@163.com

${ }^{\ddagger}$ Department Of Electronics and Information Technology,

Jiangmen Polytechnic, Jiangmen, 529000, P. R. China

Email: zhflowers@163.com

${ }^{\S}$ Beijing Institute of Technology, School of Aerospace Science Engineering,

Beijing 100081, P. R. China

Email: ruierchat@yahoo.com

'Department of Computer Science and Technology,

Tsinghua University, Beijing, 100084, P. R. China

Email: hbli@mail.tsinghua.edu.cn

Received 20 July 2011; revised 18 August 2013

We present a novel robust control scheme that deals with multi-body spacecraft attitude tracking problems. The control scheme consists of a radial basis function network (RBFN) and a robust controller. By using the finite time convergence property of the terminal sliding mode (TSM), we derive a new online learning algorithm for updating all the parameters of the RBFN that ensures the RBFN has fast approximation for the parameter uncertainties and external disturbances. We design a robust controller to compensate RBFN approximation errors and realise the anticipative stability and performance properties. We can also achieve closed-loop system stability using Lyapunov stability theory.

No detailed knowledge of the non-linear dynamics of the spacecraft is required at any point in the entire design process, and the proposed robust scheme is simple and effective and can be applied to more complex systems. Simulation results demonstrate the good tracking characteristics of the proposed control scheme in the presence of inertial uncertainties and external disturbances.

\section{Introduction}

Modern spacecraft require their attitude-control systems to provide attitude manoeuvers, tracking and high accuracy pointing in the presence of disturbances and uncertainties.

\| The work of Changqing Yuan was supported by the Natural Science Foundation of China under Grant 11372353 and 10902125 .

* The work of Hongbuo Li was supported by the National Basic Research Program of China (973 Program) under Grant 2012CB821206. 
Because of the properties of the inherent attitude dynamics, attitude tracking is a complex task. In recent decades, several control methods have been developed to treat this kind of problem. Zhang and Cao (2004) used the momentum conservation equation to propose a coordinated attitude control law for a tracking and data relay satellite. Matthew (1999) used Lyapunov stability theory to develop a Lyapunov tracking controller. However, they did not consider the mass parameter uncertainties and external disturbances. Vadali (1986) designed a sliding-mode controller based on sliding-mode control theory, which converged state trajectories to a sliding mode, though it was restricted to a simple linear spacecraft model.

$\mathrm{Wu}$ and Chen (1999) and Yang and Sun (2002) developed a mixed H2/H control method to address the spacecraft attitude tracking problem under parameter perturbation and external disturbances. However, it is difficult to apply the robust control design method to more complex models, such as multi-body spacecraft. Liang et al. (2011) presented a robust decentralised coordinated attitude control law for formation flying with external disturbances. The feasibility of applying the feedback linearisation technique to the spacecraft attitude control problem was also discussed in Sheen and Bishop (1994a). A controller for attitude control based on linearisation by coordinate transformation and non-linear feedback was presented in Bang et al. (2004) for large-angle rotational manoeuvers of spacecraft systems. However, the feedback linearisation technique requires a detailed knowledge of the non-linear dynamics. To alleviate this shortcoming, adaptive control has been applied to estimate the unknown or time-varying mass parameters. In conjunction with feedback linearisation, Sheen and Bishop (1994b) proposed a direct adaptive control law. However, the adaptive control law exhibited parameter drift and bad transient behaviours, which occurred when there was a large initial parameter estimation error (Slotine and Li 1991; Efrati 1997).

Neural networks have been shown to be the most promising technique for designing a robust, adaptive and intelligent control system. Their learning feature and powerful ability to approximate non-linear functions have been used in various applications such as pattern recognition, system identification and non-linear system modelling (Sibai et al. 2011; George 2011). In particular, neural networks have been successfully applied to many control systems, such as space platform-based manipulators, fighter aircraft, and complex spacecraft (Pazelli et al. 2011; Akpan and Hassapis 2011; Li et al. 2001; Nayeri et al. 2004). The recently developed terminal sliding mode control (TSM) enables convergence to the desired state in finite time (Feng et al. 2002; Yu and Man 2002; Yu et al. 1999). This technique has been used successfully in some control designs, such as robotic manipulators (Feng et al. 2002) and mobile target tracking (Stonier and Stonier 2004). The physical interpretation of finite time convergence lies in the fact that the TSM convergence rate grows exponentially when the state is near equilibrium.

In the current paper, we consider the non-linear dynamics of a multi-body spacecraft system and design a robust control scheme based on a radial basis function network (RBFN) and TSM. We begin by using the available information of the system, such as some nominal parameters, to weaken the non-linearity of the dynamics of the system. An RBFN controller is then designed to compensate the parameter uncertainties and external disturbances. We also develop an online fast learning algorithm for RBFN based 


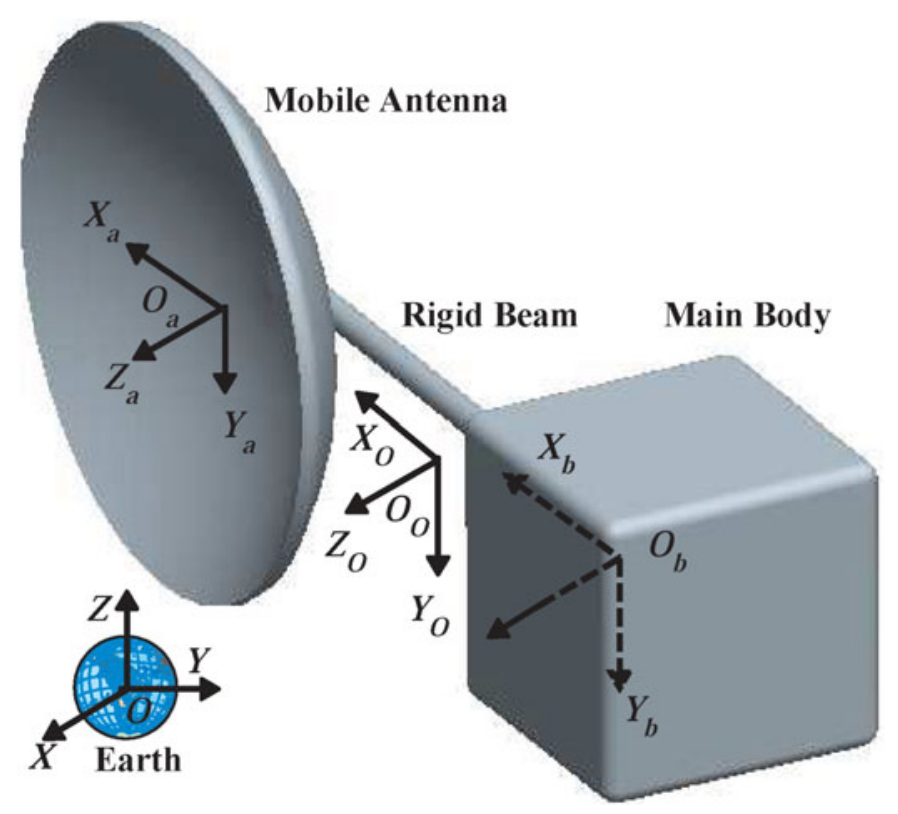

Fig. 1. (Colour online) Model of multi-body spacecraft.

on TSM. Finally, we propose a robust controller to compensate the RBFN approximation error and make the closed-loop system stable along the desired trajectory. No detailed knowledge of the non-linear plant dynamics is required at any point in the entire design process, and the proposed robust scheme is simple and effective and can be applied to more complex systems.

\subsection{Organisation of the paper}

Section 2 gives a brief description of the multi-body spacecraft system considered. In Section 3, we review the inverse system method and design a robust control scheme. In Section 4, we present and discuss the simulation results, and in Section 5, we give our conclusions. Finally, the Appendices contain definitions of the TSM controller and GCD learning algorithm, and a glossary of symbols used.

\section{Model of a multi-body spacecraft with mobile antenna}

In the current paper, we consider a multi-body spacecraft, with one large mobile antenna rotating along the axis and using reaction wheels for attitude control. The coordinate systems are shown in Figure 1. The inertial reference frame $O X Y Z$, which is fixed to the centre of the Earth, is used to determine the orbital position of the spacecraft. The orbital reference frame $O_{o} X_{o} Y_{o} Z_{o}$, which is fixed to the mass centre of the whole spacecraft, rotates about the axis $Y_{o}$. The axes of the reference frame are chosen so that the roll axis $X_{o}$ is in the flight direction, the pitch axis $Y_{o}$ is perpendicular to the orbital plane and the yaw axis $Z_{o}$ points towards the Earth. If we consider the spacecraft moving in the orbital 
plane $\left(O_{o} X_{o} Z_{o}\right)$, the dynamic equation of the multi-body spacecraft is given by

$$
M(x) \ddot{x}+Q(x, \dot{x})=T_{c}+d(t)
$$

where

$$
\begin{aligned}
& x=\left[\theta_{1} \theta_{2}\right]^{T} \\
& T_{b}=-J_{w} \omega_{w}^{\prime} \\
& T_{c}=\left[\begin{array}{c}
T_{b} \\
T_{a}
\end{array}\right] \\
& M(x)=\left[\begin{array}{cc}
J_{C} & J_{B A}^{*} \\
J_{A B}^{*} & J_{a}^{*}
\end{array}\right] \\
& Q(x, \dot{x})=\left[\begin{array}{c}
Q_{I r}+Q_{D r} \\
Q_{I a r}+Q_{D a r}
\end{array}\right] \\
& J_{C}=J_{b}+J_{w}+J_{D}+J_{a}+m_{a}\left(2 \rho_{A} r_{A} \sin \theta_{2}+r_{A}^{2}+\rho_{A}^{2}\right)-M\left(\rho_{C x}^{2}+\rho_{C z}^{2}\right) \\
& J_{B A}^{*}=J_{a}+m_{a} r_{A} \rho_{A} \sin \theta_{2}-m_{a} \rho_{A}\left(\rho_{C z} \cos \theta_{2}+\rho_{C x} \sin \theta_{2}\right) \\
& J_{A B}^{*}=J_{a}+m_{a} r_{A} \rho_{A} \sin \theta_{2}-m_{a} \rho_{A}\left(\rho_{C z} \cos \theta_{2}+\rho_{C x} \sin \theta_{2}\right) \\
& J_{a}^{*}=J_{a}-\lambda_{1} m_{a} \rho_{A}^{2} \\
& \lambda_{1}=\frac{m_{a}}{M} \\
& \lambda_{2}=\frac{m_{d}}{M} \\
& \rho_{C x}=\lambda_{1}\left(r_{\mathrm{A}}+\rho_{A} \sin \left(\theta_{2}\right)\right)+\lambda_{2} \rho_{D} \\
& \rho_{C z}=\lambda_{1} \rho_{A} \cos \left(\theta_{2}\right) \\
& Q_{I r}=\left(1+\rho_{A}\right) r_{A} m_{a} \dot{\theta}_{2} \dot{\theta}_{1} \cos \theta_{2}+m_{a} r_{A} \dot{\theta}_{2}^{2} \cos \theta_{2} \\
& Q_{D r}=m_{a} \rho_{A}\left(\rho_{C x} \cos \theta_{2}-\rho_{C z} \sin \theta_{2}\right) \dot{\theta}_{1}^{2} \\
& -\left(m_{d} \rho_{D}+m_{a} r_{A}\right)\left[\left(2 \dot{\theta}_{1} \dot{\theta}_{2}+\dot{\theta}_{2}^{2}\right) \lambda_{1} \rho_{A} \cos \theta_{2}+\dot{\theta}_{1}^{2} \rho_{C z}\right] \\
& Q_{\text {Dar }}=\left(\rho_{C x} \cos \theta_{2}-\rho_{C z} \sin \theta_{2}\right) m_{a} \rho_{A} \dot{\theta}_{1}^{2} \\
& Q_{I a r}=-m_{a} r_{A} \rho_{A} \dot{\theta}_{1}^{2} \cos \theta_{2} \text {. }
\end{aligned}
$$

\section{A robust attitude tracking controller using RBFN and TSM}

In this section we will review the inverse system method (ISM) (Hirschorn 1979) and design a robust control scheme based on RBFN and TSM.

\subsection{The inverse system method (ISM))}

The inverse system method is a commonly used control scheme for non-linear systems. The basic idea can be explained as follows (Hirschorn 1979). Consider a non-linear system

$$
\begin{aligned}
& \dot{x}=f(x, u) \\
& y=h(x, u) .
\end{aligned}
$$


Suppose the map from system input $u(t)$ to the system output $y(t)$ is $\Xi$, so

$$
y=\Xi \circ u \text {. }
$$

Now suppose there is another non-linear system, and the map from the system input $\varphi(t)$ to the output $u(t)$ is $\hat{\Xi}_{\alpha}$, so

$$
u=\hat{\Xi}_{\alpha} \circ \varphi \text {. }
$$

If there exists an $\alpha$-order derivative of y and $\hat{\Xi}_{\alpha}$ satisfying

$$
\varphi(t)=y^{\alpha}(t)
$$

and Equation (3), respectively, then the non-linear system $\hat{\Xi}_{\alpha}$ is called the $\alpha$-order integral inverse system of the non-linear system $\Xi$. We can generally realise $\hat{\Xi}_{\alpha}$ by a feedback method. Substituting Equation (3) into Equation (2), we obtain a pseudo-linear system $\Xi_{P L}$ given by

$$
y=\Xi_{P L} \circ \varphi .
$$

The relation between the input and output of the pseudo-linear system $\Xi_{P L}$ can be simply expressed by $s^{-\alpha}$. This means that the control of the non-linear system can be reasonably simplified as the control of an $\alpha$-order linear system. Hence, using linear control theory, such as LQ-optimal control theory, linear robust control theory or PID control theory, the feedback law can be designed to realise the anticipative stability and performance properties.

Now, neglecting external disturbances $d(t)$, the order integral inverse system of Equation (1) is

$$
T_{c}=M(x) \delta+Q(x, \dot{x})
$$

where $\delta$ is an auxiliary input to be determined. Substituting Equation (5) into Equation (1), we get

$$
\ddot{x}=\delta \text {. }
$$

In order to have the output $\mathrm{x}$ follow a specified trajectory, $\delta$ in Equation (6) is set to be

$$
\delta=\ddot{x}_{d}+k_{1}\left(\dot{x}_{d}-\dot{x}\right)+k_{2}\left(x_{d}-x\right)
$$

where $x_{d}$ is the desired output trajectory and $k_{1}$ and $k_{2}$ are some positive constants. Substituting Equation (7) into Equation (5), we obtain the ISM controller given by

$$
T_{c}=M\left[\ddot{x}_{d}+k_{1}\left(\dot{x}_{d}-\dot{x}\right)+k_{2}\left(x_{d}-x\right)\right]+Q .
$$

The configuration of the ISM controller is shown in Figure 2. Combining Equations (8) and (1) gives the following error dynamics:

$$
\ddot{e}+k_{1} \dot{e}+k_{2} e=0
$$

where $e=x_{d}-x$ is the output error.

Equation (9) shows that the tracking error vanishes asymptotically, and the output tends to the desired trajectory. However, in practical spacecraft systems, some perturbations in system parameters are inevitable because of the flexible structure, unmodelled dynamics, fuel slosh disturbance and the change of the orientation of solar arrays on the spacecraft. 


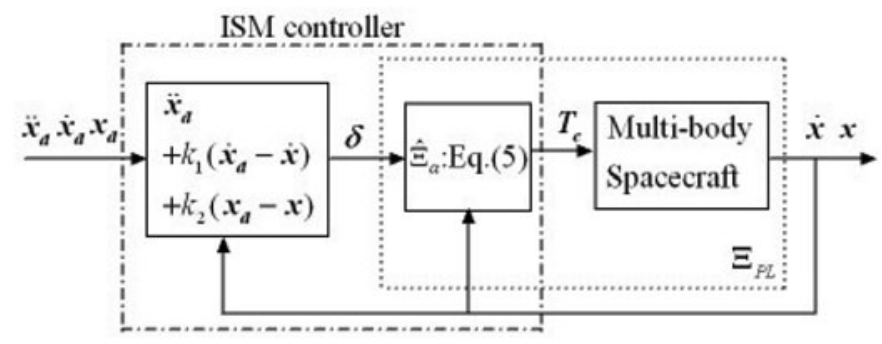

Fig. 2. Implementation of the ISM control scheme.

With perturbations of system parameters, the ISM control design cannot guarantee the performance specified by Equation (9). Moreover, the stability of the controlled system may be destroyed.

Now consider the following equivalent form of Equation (1):

$$
\ddot{x}=M_{0}^{-1}\left(T_{c}-Q_{0}\right)-\Delta
$$

where the subscript ${ }_{0}$ denotes the nominal parameter and $\Delta$ denotes the parametric uncertainty and external disturbances. In the next section we will use Equation (10) as the starting point for our proposed robust control scheme based on RBFN and TSM.

\subsection{A robust control scheme based on RBFN and TSM (TSM-RBFN RC)}

$\mathrm{Yu}$ et al. (2000) proposed a fast TSM that combines the finite time convergence property of a terminal attractor and the exponential convergence property of linear systems. In general, neural networks $(\mathrm{NN})$ are efficient for approximating highly non-linear functions within a desired accuracy (Park and Sandberg 1991; Funahashi 1998; Hornik et al. 1989). In order to achieve fast attitude tracking manoeuvers in the presence of mass parameter uncertainties and disturbances, we propose a robust control scheme combining the excellent properties of TSM and NN in the form

$$
T_{C}=M_{0}\left(u_{N N}+u_{r b}+\ddot{x}_{d}\right)+Q_{0}
$$

where $u_{N N}$ is used to compensate for the model error $\Delta$ in Equation (10). The term $u_{r b}$ is a robust controller based on TSM that compensates for NN approximation errors and realises the anticipative stability and performance properties. Moreover, through the ISM scheme, the TSM-RBFN robust control system Equation (11) makes effective use of the available information in Equation (1), such as $M_{0}$ and $Q_{0}$, to weaken the non-linearity of the system described by Equation (1). Obviously, we need to design $u_{N N}$. The configuration of the TSM-RBFN robust control system is shown in Figure 3. Substituting Equation (11) into Equation (10), we get an equivalent expression for the error dynamics in the form

$$
\ddot{e}+u_{N N}+u_{r b}-\Delta=0
$$




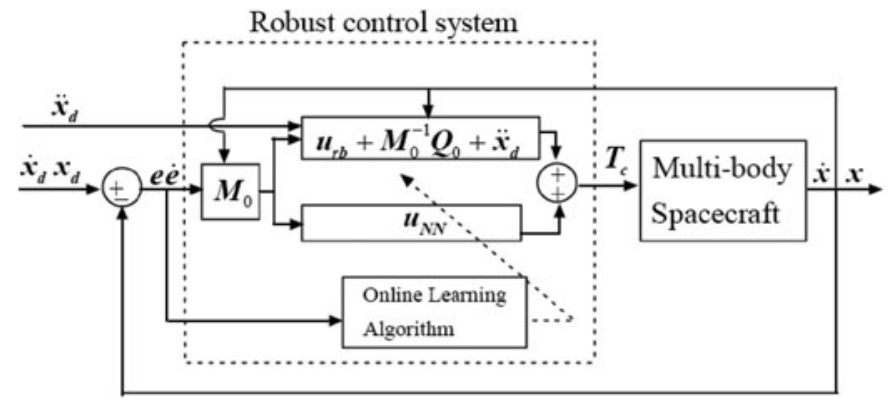

Fig. 3. Implementation of the ISM control scheme.

\subsubsection{RBFN approximation and an online learning algorithm based on TSM}

\subsubsection{RBFN architecture}

The neural scheme in Equation (11) is an RBFN consisting of a three layer processing structure. There are $n_{i}$ input nodes, $n_{k}$ hidden nodes and $n_{j}$ output nodes. It can be represented in the following form:

$$
\begin{aligned}
y & =\sum_{k=1}^{n_{k}} w_{k} \phi_{k}\left(c_{k}, \sigma_{k}, \xi\right) \\
& =W^{T} \Phi(c, \sigma, \xi)
\end{aligned}
$$

where:

- $\xi=e^{T} \in R^{n_{i} \times 1}$ is the RBFN's inputs;

- $\mathrm{y}$ is the output of RBFN;

- $w_{k}$ and $\sigma_{k} \in R^{1 \times 1}$ are the hidden-to-output layer interconnection weight, the width and centre of the Gaussian functions, respectively, which are the adjustable RBFN parameters;

- the active function used in the RBFN is chosen to be the Gaussian functions

$$
\phi_{k}=\exp \left(-\left\|e-c_{k}\right\|^{2} / \sigma_{k}^{2}\right) ;
$$

- the vector representations of $w_{k}, \sigma_{k}, c_{k}$ and $\phi_{k}$ are respectively:

$$
\begin{aligned}
W & \in R^{n_{k} \times n_{j}} \\
\sigma & \in R^{n_{k} \times 1} \\
c=\left[c_{1}^{T}, c_{2}^{T}, \cdots c_{n_{k}}^{T}\right]^{T} & \in R^{n_{k} \times 1} \\
\Phi(e)=\left[\phi_{1}, \phi_{2}, \cdots, \phi_{n_{k}}\right]^{T} & \in R^{n_{k} \times 1} .
\end{aligned}
$$

\subsubsection{RBFN approximation}

Using the results reported in Girosi and Poggio (1990) and Poggio and Girosi (1990), the model error in Equation (10) can be approximated by an RBFN through on-line learning:

$$
\Delta=W^{* T} \Phi\left(c^{*}, \sigma^{*}, \xi\right)+\varepsilon
$$


where $W^{*}, c^{*}$ and $\sigma^{*}$ are, respectively, the optimal parameter vectors (matrices) of $W, c$ and $\sigma$ in RBFN. For simplicity, we will write $\Phi^{*}$ as an abbreviation for $\Phi\left(c^{*}, \sigma^{*}, \xi\right)$ from now on. The approximation error is

$$
\varepsilon \triangleq\left(\varepsilon_{1}, \cdots, \varepsilon_{i}\right)^{\mathrm{T}} \text {. }
$$

From approximation theory, $\varepsilon_{i}$ is uniformly bounded by $\varepsilon_{i} \leqslant \gamma_{i}^{*}$, where $\gamma_{i}^{*}$ is any positive number.

The NN controller $u_{N N}$ in Equation (11) is assumed to take the form

$$
u_{N N}=\hat{W}^{T} \Phi(\hat{c}, \hat{\sigma}, \xi)
$$

where $\hat{W}, \hat{c}, \hat{\sigma}$ and $\hat{\Phi}$ are estimates of the optimal parameter vectors (matrices). For simplicity, we will write $\hat{\Phi}$ as an abbreviation for $\Phi(\hat{c}, \hat{\sigma}, \xi)$ from now on. The first partial derivatives of the function $\hat{\Phi}$ with respect to its arguments are given by

$$
\begin{aligned}
&\left.\hat{\Phi}_{c} \triangleq \frac{\partial \hat{\Phi}}{\partial \hat{c}^{T}}\right|_{(\hat{c}, \hat{\sigma})}= {\left[\begin{array}{cccc}
\frac{\partial \hat{\phi}_{1}}{\partial \hat{c}_{1}} & 0 & \cdots & 0 \\
0 & \frac{\partial \hat{\phi}_{2}}{\partial \hat{c}_{2}} & \cdots & 0 \\
\vdots & \vdots & \ddots & \vdots \\
0 & 0 & \cdots & \frac{\partial \hat{\phi}_{n_{k}}}{\partial \hat{c}_{n_{k}}}
\end{array}\right] \in R^{n_{k} \times n_{k}} } \\
&\left.\hat{\Phi}_{\sigma} \triangleq \frac{\partial \hat{\Phi}}{\partial \hat{\sigma}^{T}}\right|_{(\hat{c}, \hat{\sigma})}=\left[\begin{array}{cccc}
\frac{\partial \hat{\phi}_{1}}{\partial \hat{\sigma}_{1}} & 0 & \cdots & 0 \\
0 & \frac{\partial \hat{\phi}_{2}}{\partial \hat{\sigma}_{2}} & \cdots & 0 \\
\vdots & \vdots & \ddots & \vdots \\
0 & 0 & \cdots & \frac{\partial \hat{\phi}_{n_{k}}}{\partial \hat{\sigma}_{n_{k}}}
\end{array}\right] \in R^{n_{k} \times n_{k}}
\end{aligned}
$$

where

$$
\begin{gathered}
\frac{\partial \hat{\phi}_{k}}{\partial \hat{c}_{k}}=2 \frac{\left(e-\hat{c}_{k}\right)}{\hat{\sigma}_{k}^{2}} \hat{\phi}_{k} \in R^{n_{i} \times 1} \\
\frac{\partial \hat{\phi}_{k}}{\partial \hat{\sigma}_{k}}=2 \frac{\left\|e-\hat{c}_{k}\right\|}{\hat{\sigma}_{k}^{3}} \hat{\phi}_{k} \in R^{1 \times 1} .
\end{gathered}
$$

The Taylor series expansion of the optimal function $\Phi^{*}$ in Equation (14) about the approximation is (Nardi 2000)

$$
\Phi^{*}=\hat{\Phi}+\hat{\Phi}_{c}^{\mathrm{T}} \tilde{c}+\hat{\Phi}_{\sigma}^{\mathrm{T}} \tilde{\sigma}+\mathrm{O}^{2}(\tilde{c}, \tilde{\sigma})
$$

where

$$
\begin{aligned}
\tilde{c} \triangleq c^{*}-\hat{c} & \in R^{\left(n_{k} \times n_{i}\right) \times 1} \\
\tilde{\sigma} \triangleq \sigma^{*}-\hat{\sigma} \quad & \in R^{n_{i} \times 1} \\
\mathrm{O}^{2} & \in R^{n_{i} \times 1}
\end{aligned}
$$

We now define

$$
\tilde{W}=W^{*}-\hat{W}
$$


and consider the difference between the output of the online approximation and the optimal neural network. It can be shown that the following holds:

$$
\begin{aligned}
W^{* T} \Phi^{*}-\hat{W}^{T} \hat{\Phi}+\varepsilon & =W^{* T}\left[\hat{\Phi}+\hat{\Phi}_{c}^{\mathrm{T}} \tilde{c}+\hat{\Phi}_{\sigma}^{\mathrm{T}} \tilde{\sigma}+\mathrm{O}^{2}(\tilde{c}, \tilde{\sigma})\right]-\hat{W}^{T} \hat{\Phi}+\varepsilon \\
& =\tilde{W}^{T} \hat{\Phi}+\hat{W}^{T} \hat{\Phi}_{c}^{\mathrm{T}} \tilde{c}+\hat{W}^{T} \hat{\Phi}_{\sigma}^{\mathrm{T}} \tilde{\sigma}+E_{r}
\end{aligned}
$$

where

$$
E_{r} \triangleq\left(\bar{\varepsilon}_{1}, \cdots, \bar{\varepsilon}_{i}\right)^{T}=\tilde{W}^{T}\left(\hat{\Phi}_{c}^{\mathrm{T}} \tilde{c}+\hat{\Phi}_{\sigma}^{\mathrm{T}} \tilde{\sigma}\right)+W^{* T} \mathrm{O}^{2}(\tilde{c}, \tilde{\sigma})+\varepsilon
$$

is the higher order term. From the results in Nardi (2000), $E_{r}$ is bounded by the inequality (17) where $v_{i}$ is a positive number:

$$
\left|\bar{\varepsilon}_{i}\right|<v_{i}
$$

\subsubsection{Online learning algorithm based on TSM (TSM-OLA)}

We use an online learning algorithm, which guarantees that the NN controller can deal with unexpected situations quite well. In practical online applications, the algorithm must have a high convergence speed. However, a simple standard gradient descent learning algorithm can be very slow when the approximation error is very small. In order to increase the convergence speed, we propose an online learning algorithm based on TSM (TSM-OLA) as follows. The TSM (Yu et al. 2000) can be described by

$$
S \triangleq\left(s_{1}, \cdots, s_{i}\right)^{\mathrm{T}}=\dot{e}+\alpha e+\beta e^{\gamma}
$$

where

$$
\begin{aligned}
& \alpha=\operatorname{diag}\left(a_{1} \cdots a_{i}\right) \\
& \beta=\operatorname{diag}\left(b_{1} \cdots b_{i}\right)
\end{aligned}
$$

are the design matrices $\left(a_{i}>0, b_{i}>0\right)$ and $\gamma \triangleq q / p$, where $p$ and $q$, with $q<p$, are positive odd integers. The TSM-OLA can then be described by

$$
\begin{aligned}
\dot{\hat{W}} & =\eta_{1} \hat{\Phi} \cdot S^{T} \\
\dot{A}_{c} & =\eta_{2} S^{T} \cdot \hat{W}^{T} \cdot \hat{\Phi}_{c}^{T} \\
\dot{\hat{\sigma}}^{T} & =\eta_{3} S^{T} \cdot \hat{W}^{T} \cdot \hat{\Phi}_{\sigma}^{T}
\end{aligned}
$$

where $\eta_{1}, \eta_{2}$ and $\eta_{3}$ are positive constants. In the TSM-OLA, the introduction of the nonlinear item $\operatorname{diag}\left(e^{\gamma}\right)$ amplifies the approximation error's contribution to the convergence rate in the vicinity of $e=0$.

Because the real implementation has to be carried out in a discrete-time framework, the TSM-OLA can be converted into a discrete form:

$$
\begin{aligned}
& \hat{W}(t+1)=\hat{W}(t)+\eta_{1} \hat{\Phi}(t) \cdot S(t)^{T}+\mu_{1}(\hat{W}(t)-\hat{W}(t-1)) \\
& \hat{c}(t+1)^{T}=\hat{c}(t)^{T}+\eta_{2} S(t)^{T} \cdot \hat{W}(t)^{T} \cdot \hat{\Phi}_{c}(t)^{T}+\mu_{2}\left(\hat{c}(t)^{T}-\hat{c}(t-1)^{T}\right) \\
& \hat{\sigma}(t+1)^{T}=\hat{\sigma}(t)^{T}+\eta_{3} S(t)^{T} \cdot \hat{W}(t)^{T} \cdot \hat{\Phi}_{\sigma}(t)^{T}+\mu_{3}\left(\hat{\sigma}(t)^{T}-\hat{\sigma}(t-1)^{T}\right)
\end{aligned}
$$


where

$$
0 \leqslant \mu_{i}<1 \quad(i=1,2,3)
$$

is a fixed gain and

$$
\begin{aligned}
& (\hat{W}(t)-\hat{W}(t-1)) \\
& \left(\hat{c}(t)^{T}-\hat{c}(t-1)^{T}\right) \\
& \left(\hat{\sigma}(t)^{T}-\hat{\sigma}(t-1)^{T}\right)
\end{aligned}
$$

are the momentum terms, which tend to damp oscillations in the parameter vector and keep the parameter vector moving in the correct direction.

3.2.2. Robust control scheme and stability analysis

\subsubsection{Robust control scheme}

Using the results in Section 3.2.1, we can obtain the TSM-RBFN robust control system as follows. Consider the multi-body spacecraft dynamics represented by Equation (1). If the TSM-RBFN robust control system is given by Equation (11), where the NN controller is given by Equation (15), the robust controller is given by Equation (22) and the TSMOLA of the TSM-RBFN robust control system is given by Equations (19)-(21), then the closed-loop system is Lyapunov stable and the tracking error will converge to zero in finite time. We have

$$
u_{r b}=\alpha \dot{e}+\gamma \beta \operatorname{diag}\left(e^{\gamma-1}\right) \dot{e}+v \operatorname{sign}(S)
$$

where

$$
v=\operatorname{diag}\left(v_{1}, v_{2}\right),
$$

is the bound of $\bar{\varepsilon}_{i}$, which satisfies the inequality (17) for $i=1,2$.

\subsubsection{Stability analysis}

We choose the following Lyapunov function candidate:

$$
V=\frac{1}{2} S^{T} S+\frac{1}{2 \eta_{1}} \operatorname{tr}\left(\tilde{W}^{T} \tilde{W}\right)+\frac{1}{2 \eta_{2}} \tilde{c}^{T} \tilde{c}+\frac{1}{2 \eta_{3}} \tilde{\sigma}^{T} \tilde{\sigma},
$$

where $\operatorname{tr}(\cdot)$ is the trace operator. Differentiating Equation (23) along the trajectories of Equation (12), we get

$$
\dot{V}=S^{T} \dot{S}-\frac{1}{\eta_{1}} \operatorname{tr}\left(\tilde{W}^{T} \dot{\hat{W}}\right)-\frac{1}{\eta_{2}} \dot{\hat{c}}^{T} \tilde{c}-\frac{1}{\eta_{3}} \dot{\hat{\sigma}}^{T} \tilde{\sigma} .
$$

The first derivative of Equation (18) is

$$
\dot{S}=\ddot{e}+\alpha \dot{e}+\gamma \beta \operatorname{diag}\left(e^{\gamma-1}\right) \dot{e} .
$$


Substituting Equation (25) into Equation (24), and using Equations (12), (14), (15), (16) and (22), we get

$$
\begin{aligned}
\dot{V}= & S^{T}\left(\tilde{W}^{T} \hat{\Phi}+\hat{W}^{T} \hat{\Phi}_{c}^{\mathrm{T}} \tilde{c}+\hat{W}^{T} \hat{\Phi}_{\sigma}^{\mathrm{T}} \tilde{\sigma}+E_{r}-v \operatorname{sign}(S)\right) \\
& -\frac{1}{\eta_{1}} \operatorname{tr}\left(\tilde{W}^{T} \dot{\hat{W}}\right)-\frac{1}{\eta_{2}} \dot{\hat{c}}^{T} \tilde{c}-\frac{1}{\eta_{3}} \dot{\hat{\sigma}}^{T} \tilde{\sigma} \\
= & \operatorname{tr}\left[\tilde{W}^{T}\left(\frac{p}{q} \hat{\Phi} \cdot S^{T} \cdot B \cdot \operatorname{diag}\left(\dot{e}^{p / q-1}\right)-\frac{1}{\eta_{1}} \dot{\hat{W}}\right)\right] \\
& +\left(\frac{p}{q} S^{T} \cdot B \cdot \operatorname{diag}\left(\dot{e}^{p / q-1}\right) \cdot \hat{W}^{T} \cdot \hat{\Phi}_{c}^{T}-\frac{1}{\eta_{2}} \dot{\hat{c}}^{T}\right) \tilde{c} \\
& +\left(\frac{p}{q} S^{T} \cdot B \cdot \operatorname{diag}\left(\dot{e}^{p / q-1}\right) \cdot \hat{W}^{T} \cdot \hat{\Phi}_{\sigma}^{T}-\frac{1}{\eta_{3}} \dot{\hat{\sigma}}^{T}\right) \tilde{\sigma} \\
& +S^{T}\left(E_{r}-v \operatorname{sign}(S)\right) .
\end{aligned}
$$

If $\dot{\hat{W}}, \dot{\hat{c}}^{T}$ and $\dot{\hat{\sigma}}^{T}$ are given by Equations (19), (20) and (21), respectively, Equation (26) can be rewritten as

$$
\begin{aligned}
\dot{V} & =-S^{T}\left(v \operatorname{sign}(S)-E_{r}\right) \\
& \leqslant-\sum_{i=1}^{2}\left(\kappa_{i}\left|s_{i}\right|\right)<0 \quad \text { for } s_{i} \neq 0
\end{aligned}
$$

where

$$
\kappa_{i}=v_{i}-|\bar{\varepsilon}|>0 .
$$

Equation (27) means that the FTSM manifold $s_{i}(i=1,2)$ converges to zero. On the other hand, in the TSM $s_{i}=0(i=1,2)$, and the system state will reach zero in finite time (Yu et al. 2000).

Remark 3.1. In order to eliminate chattering, the signum function of Equation (22) is often replaced by a continuous saturation function

$$
\operatorname{sat}\left(s_{i}, \varepsilon\right)=\left\{\begin{array}{ll}
s_{i} / \varepsilon & \text { if }\left|s_{i}\right|<\varepsilon \\
\operatorname{sign}\left(s_{i}\right) & \text { if }\left|s_{i}\right| \geqslant \varepsilon
\end{array} \quad(i=1,2)\right.
$$

where $\varepsilon>0$ is the width of the boundary layer. Equation (22) can now be rewritten to give

$$
u_{r b}=\alpha \dot{e}+\gamma \beta \operatorname{diag}\left(e^{\gamma-1}\right) \dot{e}+v \cdot \operatorname{sat}(S, \varepsilon)
$$

where

$$
\operatorname{sat}(S, \varepsilon)=\left[\operatorname{sat}\left(s_{1}, \varepsilon\right), \operatorname{sat}\left(s_{2}, \varepsilon\right)\right]^{\mathrm{T}}
$$

Remark 3.2. There exists a possible singularity in the TSM-RBFN robust controller as $e \rightarrow 0$. Since $e=0$ only approaches along a sliding mode, we observe that for a general choice of $\gamma$, we have

$$
\dot{e}=-\alpha e-\beta \operatorname{diag}\left(e^{\gamma}\right)
$$


Table 1. Parameter values for the model (international units)

\begin{tabular}{lccc}
\hline \hline Parameter & $J_{w}$ & $J_{D}$ & $J_{a}$ \\
Value & 0.0796 & 30.0 & 40.0 \\
Parameter & $\rho_{A}$ & $\rho_{D}$ & $r_{A}$. \\
Value & 0.25 & 0.40 & 0.60 \\
Parameter & $m_{d}$ & $M$ & $m_{a}$ \\
Value & 0.50 & 261.4 & 10.0 \\
\hline
\end{tabular}

while sliding, and that the component in Equation (22) is

$$
\gamma \beta \operatorname{diag}\left(\mathrm{e}^{\gamma-1}\right) \dot{\mathrm{e}} \rightarrow-\gamma \beta\left(\alpha \mathrm{e}^{\gamma}+\beta \mathrm{e}^{2 \gamma-1}\right) .
$$

Consequently, there will be a singularity in Equation (22) unless $\gamma$ is chosen so that $2 \gamma>1$. To satisfy this requirement, we set $\gamma=3 / 5$ for the following examples.

\section{Simulation results}

Numerical simulations were performed to assess the closed-loop stability performance of the proposed TSM-RBFN robust control system in the presence of inertial uncertainties and external disturbances. We used Simulink as the simulation environment for our simulations and ODE45 to perform the numerical integration. Relative and absolute error tolerances were uniformly set to be $1 \mathrm{e}-6$ throughout the simulations. The initial values $\theta_{10}$ and $\theta_{20}$ of $\theta_{1}$ and $\theta_{2}$ were set to 0.001 , and the nominal value of $J_{b}$ was set to 50.00 .

During the simulation, the inertia of the the main body was changed as follows. The actual inertia $J_{b}$ begins with $120 \%$ of the nominal values at $t=0$ second and the inertia is gradually increased to $140 \%$ of the nominal values from 0 second to 10 seconds. The inertia is then suddenly decreased to $100 \%$ of the nominal values at 10 seconds. The decreased inertia is constant until $\mathrm{t}=15$ seconds at which the inertia is suddenly increased to $160 \%$ of the nominal values. This increased value is then maintained until the end of simulation. The model parameters are summarised in Table 1.

The external disturbance torque is given by

$$
d(t)=\left[\begin{array}{l}
0.10 \sin (10 \mathrm{t}) \\
0.05 \sin (10 \mathrm{t})
\end{array}\right] .
$$

In addition, the parameters of the TSM-RBFN robust control system were set to the values given in Table 2 .

All the parameters of the TSM-RBFN robust control system were chosen to achieve the best control performance in the simulation. The choice of network size is not an easy task and corresponds to the usual model order determination problem (Lin and Wai 1998; Passino 2004). The RBFN was set to have two, ten and two neurons at the input, hidden and output layers, respectively. Each of the centres and the width of the 
Table 2. Parameter values for TSM-RBFN RC

\begin{tabular}{lccccl}
\hline \hline Parameter & $a_{1}$ & $a_{2}$ & $\eta_{1}$ & $\eta_{2}$ & $\eta_{3}$ \\
Value & 2.0 & 2.0 & 0.12 & 0.10 & 0.10 \\
Parameter & $b_{1}$ & $b_{2}$ & $\mu_{1}$ & $\mu_{2}$ & $\mu_{3}$ \\
Value & 1.0 & 1.0 & 0.05 & 0.05 & 0.05 \\
Parameter & $v_{1}$ & $v_{2}$ & $\gamma$ & & \\
Value & 1.0 & 1.5 & $3 / 5$ & & \\
\hline
\end{tabular}

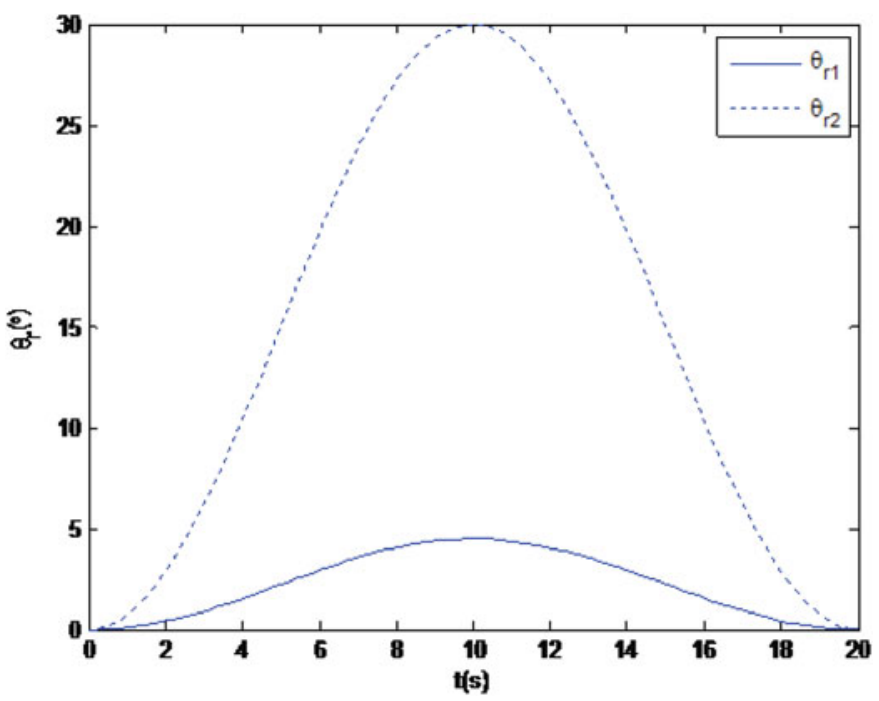

Fig. 4. (Colour online) Desired attitude of main body and mobile antenna.

Gaussian functions were initialised with arbitrary numbers, and each of the weights of the output layer with zeros.

To compare the robustness against inertial uncertainties and external disturbances, four control methods were considered:

- an ISM controller (Equation (8));

- a TSM controller (Equation (30) - see Appendix A);

- a GGD-RBFN robust control scheme (Equations (11), (31), (32) and (33)); and

- the TSM-RBFN robust control scheme (Equations (11), (19), (20) and (21)).

The simulation results are shown in Figures 4-10. The desired attitudes of the main body and the antenna are shown in Figure 4. The tracking errors $e_{1}$ and $e_{2}$ are shown in Figures 5-6, and the tracking errors when the general gradient descent learning algorithm (Equations (31-33) - see Appendix A) was applied to update all parameters of the RBFN in (11) are show as dotted lines in Figures 5-6.

The control torques with the TSM-RBFN robust control law are shown in Figure 7. The output $u_{N N}$ of the RBFN whose the parameters are updated by TSM-OLA is shown in Figure 8. It is clear from Figure 8 that the RBFN can quickly compensate for the parameter uncertainties and external disturbances, and the robust controller can 


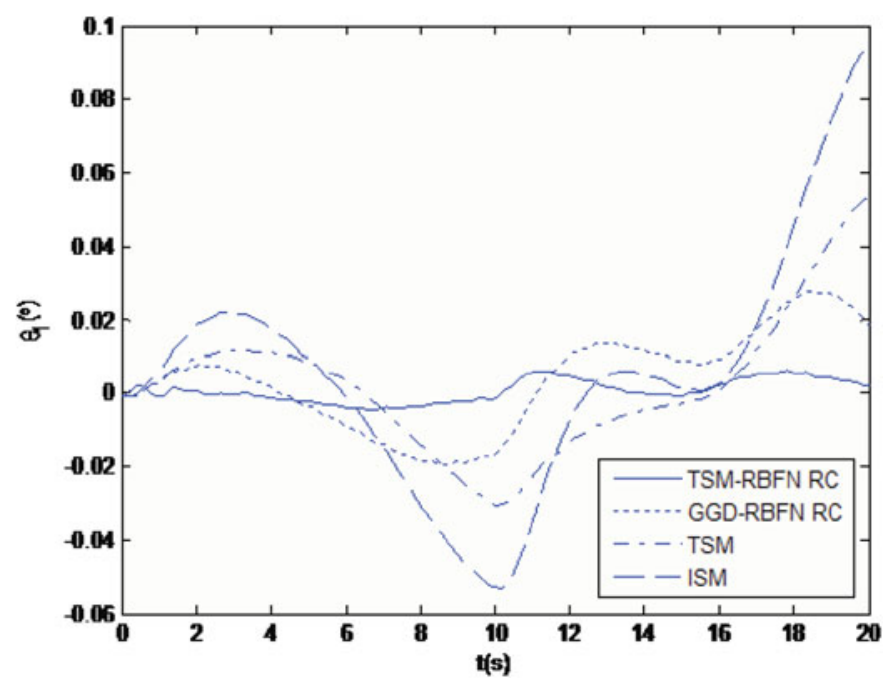

Fig. 5. (Colour online) Tracking error of the main body.

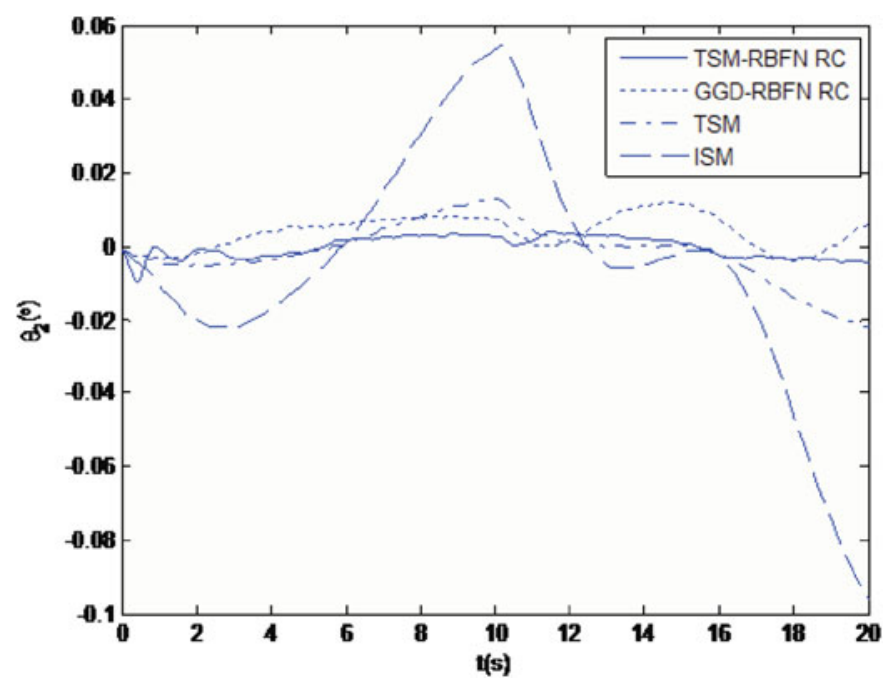

Fig. 6. (Colour online) Tracking error of the mobile antenna.

compensate for the RBFN approximation error and make the closed-loop system stable along the desired trajectory. The mean square errors and the maximum errors of the attitude tracking are shown in Figures 9-10. The results of the four proposed methods show that the TSM-RBFN robust control scheme is better at reducing the effects of parameter perturbations and external disturbances. This is because the TSM-RBFN robust control scheme is added in parallel to learn the system's model error $\Delta$. 


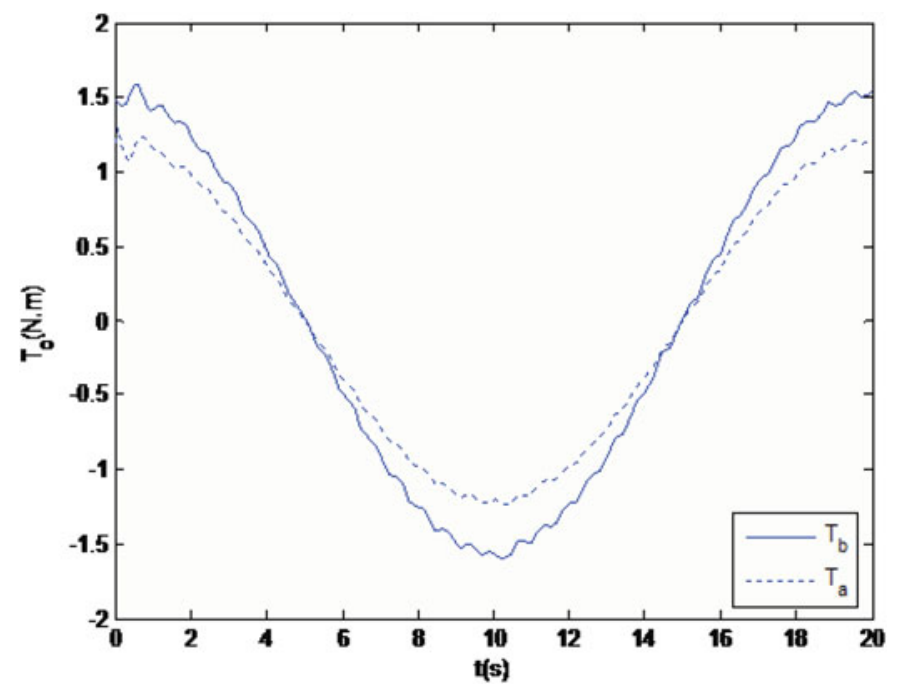

Fig. 7. (Colour online) Control torques.

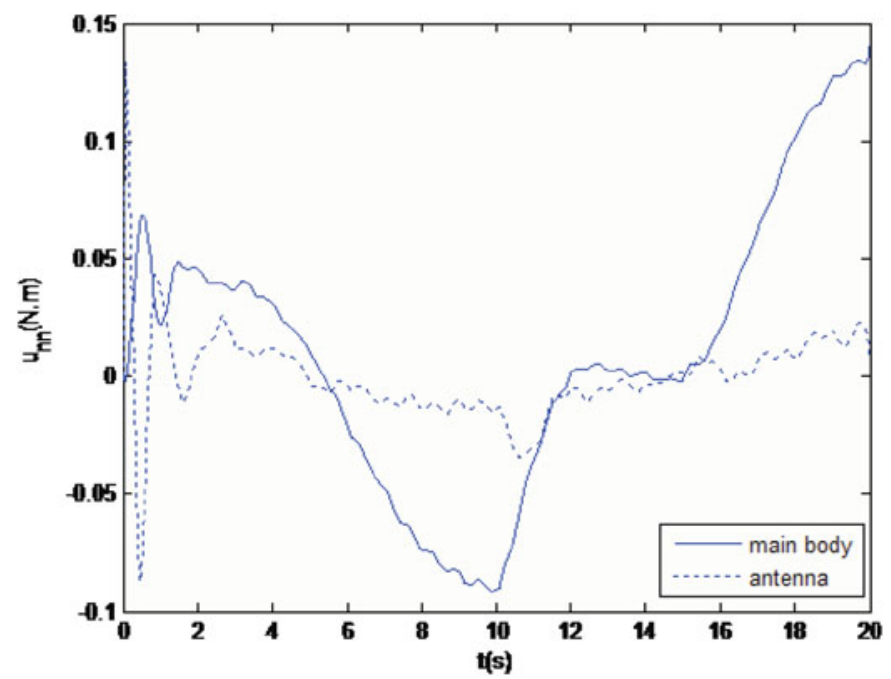

Fig. 8. (Colour online) The output of the RBFN.

\section{Conclusions}

Since the dynamic equations of the multi-body spacecraft are highly non-linear, and it is difficult to obtain detailed knowledge of the non-linear dynamics, we have proposed a robust control scheme for multi-body spacecraft attitude tracking in the presence of mass parameter uncertainties and external disturbances. The control scheme consists of an RBFN and a robust controller. The RBFN enables us to compensate for the parameter uncertainties and external disturbances, and the robust controller enables us to 


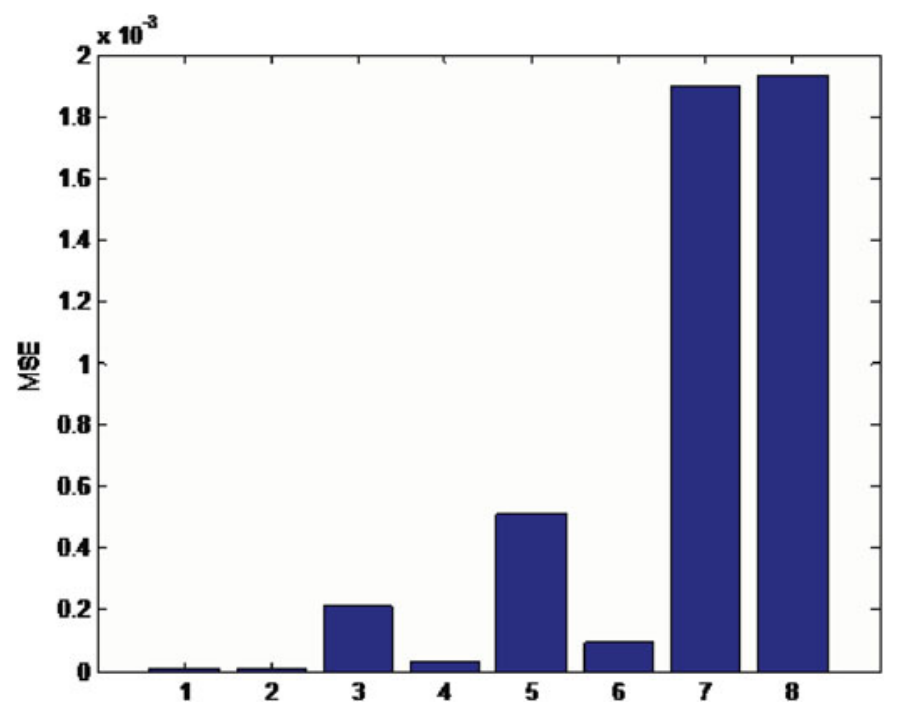

Fig. 9. (Colour online) The mean square error of the attitude tracking.

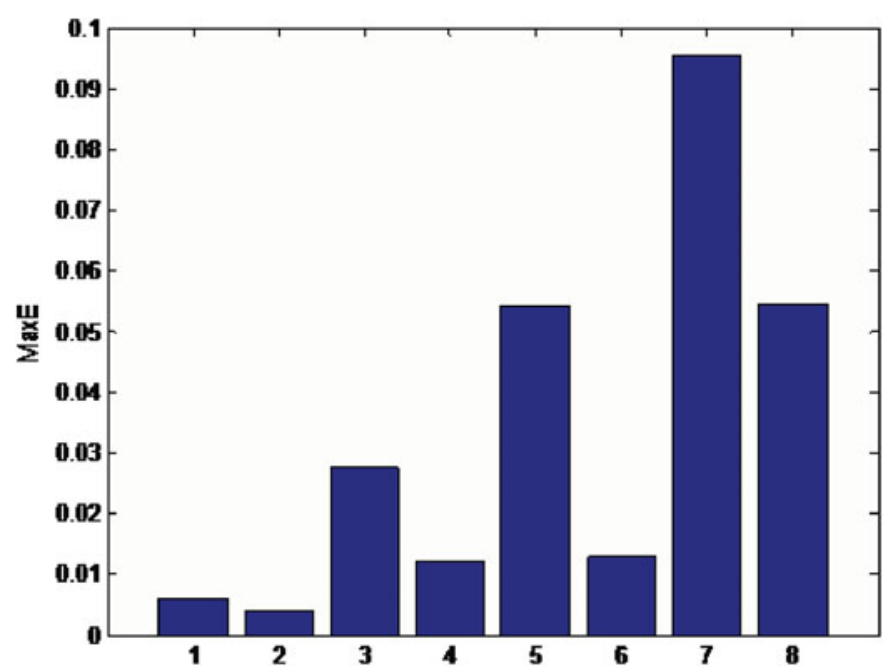

Fig. 10. (Colour online) The maximum value of the attitude tracking error.

compensate for the RBFN approximation error and makes the closed-loop system stable along the desired trajectory. In order to increase the RBFN convergence speed, we have also proposed a new online learning algorithm (TSM-OLA) in which the introduction of the non-linear item improves the efficiency of the artificial neural network. Closed-loop system stability can also be achieved on the basis of Lyapunov stability theory.

No detailed knowledge of the non-linear dynamics is required in any part of the design process. Moreover, the proposed robust scheme is simple and can be applied to 
more complex systems. The simulation results for a multi-body spacecraft show that the proposed robust control scheme exhibits significant advantages for the attitude tracking control of a spacecraft in the presence of mass parameter uncertainties and external disturbances. This NN-based robust control system has the potential to enable autonomous operations of on-orbit multi-body spacecraft.

\section{Appendix A. TSM controller}

The TSM controller is based on $\mathrm{Yu}$ et al. (2000) and given by

$$
T_{C}=M_{0}\left(\ddot{x}_{d}+\alpha \dot{e}+\gamma \beta \operatorname{diag}\left(|e|^{\gamma-1}\right) \dot{e}+v \operatorname{sat}(S, \varepsilon)\right)+Q_{0} .
$$

\section{Appendix B. The GGD learning algorithm}

The GGD learning algorithm is described by the following equations:

$$
\begin{aligned}
& \dot{\hat{W}}=\eta_{1} \hat{\Phi} \cdot e^{T} \\
& \dot{\hat{c}}^{T}=\eta_{2} e^{T} \cdot \hat{W}^{T} \cdot \hat{\Phi}_{c}^{T} \\
& \dot{\hat{\sigma}}^{T}=\eta_{3} e^{T} \cdot \hat{W}^{T} \cdot \hat{\Phi}_{\sigma}^{T} .
\end{aligned}
$$

\section{Appendix C. Glossary of symbols}

$\begin{array}{ll}\theta_{1} & =\text { attitude angles of the main body } \\ \theta_{2} & =\text { rotational angles of the mobile antenna } \\ J_{w} & =\text { moment of inertia of the reaction wheels } \\ J_{b} & =\text { moment of inertia of the main body about the axis } \\ J_{D} & =\text { moment of inertia of the mobile antenna about the axis } \\ J_{a} & =\text { control torque of the main body } \\ T_{b} & =\text { distance from point } O_{a} \text { to point } O_{b} \\ r_{A} & =\text { distance from the mass centre of the rigid beam to } O_{b} \\ \rho_{C} & =\text { distance from the mass centre of the mobile antenna to } O_{a} \\ \rho_{D} & =\text { Earth centre inertial frame } \\ \rho_{A} & =\text { orbital reference frame } \\ O X Y Z & \text { reference coordinates of the main body } \\ O_{o} X_{o} Y_{o} Z_{o} & \text { reference coordinates of the mobile antenna } \\ O_{b} X_{b} Y_{b} Z_{b} & =\text { external disturbance torque } \\ O_{a} X_{a} Y_{a} Z_{a} & =\text { angular velocity of the reaction wheels } \\ d & =\text { control torque of the mobile antenna } \\ \omega_{w} & =\text { mass of the rigid beam } \\ T_{a} & =\text { mass of the system } \\ m_{d} & =\text { mass of the mobile antenna. } \\ M & \end{array}$




\section{Acknowledgments}

The authors would like to thank Professor Deng Zhidong of the Department of computer science and Technology, Tsinghua University, for many helpful discussions on the subject of this paper.

\section{References}

Akpan, V.A. and Hassapis, G. D. (2011) Nonlinear model identification and adaptive model predictive control using neural networks. ISA Transactions 50 (2) 177-194.

Bang, H., Lee, J.-S. and Eun, Y.-J. (2004) Nonlinear Attitude Control for a Rigid Spacecraft by feedback linearization. KSME International Journal 18 (2) 203-210.

Efrati, T. (1997) Tracking Control of Mechanical Systems Using Artificial Neural Networks, Ph.D. Thesis, USC.

Feng, Y., Yu, X. and Man, Z. (2002) Non-singular terminal sliding mode control of rigid manipulators. Automatica 38 (12) 2159-2167.

Funahashi, K. (1998) On the Approximate Realization of Continuous Mappings by Neural Networks. Neural Networks 2 (3) 183-192.

George A. (2011) Multivariate sigmoidal neural network approximation. Neural Networks 24 (4) $378-386$.

Girosi, F. and Poggio, T. (1990) Networks and the best approximation property. Biological Cybernetics 63 (3) 169-176

Hirschorn, R. M. (1979) Invertibility of multivariable nonlinear control systems. IEEE Transactions on Automatic Control 24 (8) 855-865.

Hornik, K., Stinchcombe, M and White, H. (1989) Multilayer Feedforword Networks are Universal Approximators. Neural Networks 2 359-366.

Li, Y., Sundararajan, N. and Saratchandran, P. (2001) Neuro-controller design for nonlinear fighter aircraft maneuver using fully tuned RBF networks. Automatica 37 (8) 1293-1301.

Liang, H., Sun, Z. and Wang, J. (2011) Robust decentralized coordinated attitude control of spacecraft formation. Acta Astronautica 69 (5-6) 280-288.

Lin, F. J. and Wai, R. J. (1998) Ultrasonic motor servo drive with on-line trained neural network model-following controller. IEEE Proceedings, Electric Power Applications 145 (2) 105-110.

Matthew, R. L. (1999) Spacecraft Attitude Tracking Control, M.Sc. Thesis, The Virginia Polyechnic Institute and State University.

Nardi, F. (2000) Neural network based adaptive algorithms for nonlinear control, Ph.D. Thesis, The School of Aerospace Engineering, Georgia Institute of Technology.

Nayeri, M. R. D., Alasty, A. and Daneshjou, K. (2004) Neural optimal control of flexible spacecraft slew maneuver. Acta Astronautica 55 (10) 817-827.

Park, J. and Sandberg, I. W. (1991) Universal approximation using radial basis function networks. Neural Computation 3 (2) 246-257.

Passino, K. M. (2004) Biomimicry for Optimization, Control, and Automation, Springer-Verlag.

Pazelli, T. F. P. A. T., Terra, M. H. and Siqueira, A. A. G. (2011) Experimental investigation on adaptive robust controller designs applied to a free-floating space manipulator. Control Engineering Practice 19 (4) 395-408.

Poggio, T. and Girosi, F. (1990) Networks for approximation and learning. Proceedings of the IEEE 78 (9) 1481-1497.

Sheen, J.J. and Bishop, R.H. (1994a) Spacecraft Nonlinear Control. Journal of the Astronautical Sciences 42 (3) 361-377. 
Sheen, J. J. and Bishop, R.H. (1994b) Adaptive Nonlinear Control of Spacecraft. Journal of the Astronautical Sciences 42 (4) 451-472.

Sibai, F. N., Hosani, H. I., Naqbi, R. M., Dhanhani, S. and Shehhi, S. (2011) Iris recognition using artificial neural networks. Expert Systems with Applications 38 (5) 5940-5946.

Slotine, J.S. and Li, W. (1991) Applied Nonlinear Control, Prentice Hall.

Stonier, D. J. and Stonier, R. J. (2004) Obstacle avoidance and finite-time tracking of mobile targets. In: 2nd international conference on autonomous robots and agents, Palmerston North, New Zealand $58-63$.

Vadali, S. R. (1986) Variable-structure control of spacecraft large-angle maneuvers. Journal of Guidance, Control, and Dynamics 9 (2) 235-239.

Wu, C. S. and Chen, B. S. (1999) Unified Design for $H_{2}, H_{\infty}$, and Mixed control of spacecraft. Journal of Guidance, Control, and Dynamics 22 (6) 854-896.

Yang, C. D. and Sun, Y.P. (2002) Mixed $H_{2} / H_{\infty}$ state-feedback design for microsatellite attitude control. Control Engineering Practice 10 (9) 951-970.

Yu, X. and Man Z. (2002) Variable structure systems with terminal sliding modes. Springer-Verlag Lecture Notes in Control and Information Sciences 274 109-128.

Yu, X., Wu, Y. and Man Z. (1999) On global stabilization of nonlinear dynamical systems. SpringerVerlag Lecture Notes in Control and Information Sciences 247 109-122.

$\mathrm{Yu}, \mathrm{S}$., Yu, X. and Zhihong, M. (2000) Robust global terminal sliding mode control of SISO nonlinear uncertain systems. Proceedings of the 39th IEEE conference on decision and control.

Zhang, S. J. and Cao, X. C. (2004) Coordinated attitude control for a tracking and data relay satellite with mobile antennas. Aircraft Engineering and Aerospace Technology 76 (4) 414-419. 